\title{
SALUD Y POBLACIÓN LGBTI+ EN CHILE: DESDE LA INVISIBILIDAD A UN ENFOQUE IDENTITARIO
}

Caterine Galaz*

Catalina Álvarez Martinez-Conde

Rodolfo Morrison

\section{Resumen}

Las políticas públicas sobre las sexualidades LGBTI+ no heterosexuales en Chile han pasado de una invisibilización intencionada hasta un reciente posicionamiento identitario. Luego de la dictadura, la categoría de la "diversidad sexual" en salud se ha ido posicionando como un derecho, a la vez que estableciendo regulaciones respecto a cuáles son las prácticas legitimadas de ser/hacer de las personas LGBTI+. A través de un análisis discursivo de normativas, planes y directrices de salud, se analiza históricamente cómo se han ido sedimentando en esta área las visiones sobre la orientación sexual e identidad de género desde el fin de la dictadura (1990) hasta la actualidad. Se condensan tres formas de actuación en el tiempo: a) la invisibilidad como norma o la "política de la no política"; b) una construcción problemática de la orientación sexual e identidad de género; y c) la sobreespecificación identitaria de la política pública en salud.

Palabras clave: Identidad de género, conducta sexual, política pública, salud, diversidad sexual.

Facultad de Ciencias Sociales de la Universidad de Chile, Comuna de Ñuñoa, Santiago de Chile, Chile.

Dirección postal: Facultad de Ciencias Sociales, Universidad de Chile, Av. Capitán Ignacio Carrera Pinto No1045, Nuñoa, Santiago de Chile, Chile.

Correo electrónico: cgalazvalderrama@uchile.cl

** Universidad Autónoma de Barcelona; Departamento de Psicología Social - 08193 Bellaterra, Barcelona, España.

Dirección postal: Edificio B, 08193 Bellaterra, Barcelona, España.

Correo electrónico: cata.alvarezmc@gmail.com

**** Universidad de Chile, Independencia, Santiago de Chile.

Dirección postal: Depto. de Terapia Ocupacional y Ciencia de la Ocupación, Facultad de Medicina, Universidad de Chile, Independencia 1027, Independencia, Santiago de Chile.

Correo electrónico: rodolfo.morrison@uchile.cl 


\section{Resumo}

Saúde e população LGBTI + no Chile: da invisibilidade a uma abordagem identitária

As políticas públicas sobre sexualidades não heterossexuais no Chile passaram de uma invisibilidade intencional a um posicionamento identitário recente. Após a ditadura, a categoria "diversidade sexual" em saúde foi-se estabelecendo como um direito, ao mesmo tempo que se regulamentaram as práticas legítimas de ser/fazer das pessoas LGBTI+. Por meio de uma análise discursiva das normas, planos e diretrizes de saúde, analisa-se historicamente como se foram sedimentando nesta área as visões sobre orientação sexual e identidade de género no Chile desde o fim da ditadura (1990) até o presente momento. Emergem três formas de ação ao longo do tempo: a) a invisibilidade como norma ou a "política da não política"; b) uma construção problemática da orientação sexual e identidade de género; e c) a sobre-especificação identitária nas políticas públicas de saúde.

Palavras-chave: Identidade de género, comportamento sexual, políticas públicas, saúde, diversidade sexual.

Abstract

Health and LGBTI+ People in Chile: From invisibility to an identity-based approach

Public policies on non-heterosexual sexualities in Chile have shifted from an intentional invisibility to a recent focus on the identity of LGBTI+ people. After the dictatorship, the category of "sexual diversity" in health became gradually acknowledged as a right, at the same time that regulations were established about legitimate practices of making/being for LGBTI+ people. Through a discourse analysis of health regulations, plans and guidelines, we present an historical analysis of the way in which views about sexual orientation and gender identity have evolved in this area from the end of the dictatorship (1990) to the present. Three forms of action have emerged over time: a) invisibility as a norm or "policy of non-policy"; b) a problematic construction of sexual orientation and gender identity; c) identity overspecification of public health policies.

Keywords: Gender identity, sexual behaviour, public policy, health, sexual diversity.

\section{Introducción y antecedentes}

Las políticas públicas sobre sexualidades $\mathrm{LGBTI}+{ }^{1}$ en Chile han pasado de una invisibilización intencionada hasta un reciente posicionamiento identitario. Esto se relaciona con las luchas de diferentes colectivos sociales en el país y con el avance de las discusiones sobre derechos sexuales en la región latinoamericana (Galaz et al. 2018). Esta situación es relevante, pues la persecución histórica hacia

Sobre esta categoría existe discusión (Pecheny 2005; Iosa y Rabbia 2011; Esguerra y Bello 2014). Adoptamos esta forma, que incluye el signo más (+) como guiño de apertura, para ubicar al lector/a en el campo temático, considerando las diversas orientaciones e identidades sexuales que las personas pueden adscribirse. En ocasiones hablaremos de personas no heterosexuales para aludir a una problemática central que atraviesa a estas subjetividades. Finalmente utilizaremos el término "diversidad sexual" cuando hablemos de las políticas públicas en Chile porque ha sido el concepto aglutinante con que se ha trabajado (Galaz et. al 2018). 
el colectivo de personas LGBTI+ en dictadura fue una práctica habitual, ya que atentaba contra el orden y una idea de ciudadanía heterosexual (Garrido 2016). El área de salud fue una de las primeras en generar acciones públicas en la materia (Galaz et al. 2018). Así, la categoría de diversidad sexual se ha ido posicionando como un derecho, pero a la vez, ha ido estableciendo regulaciones respecto de cuáles son las prácticas legitimadas de ser/hacer de las personas LGBTI+. Los últimos estudios que han analizado la cuestión de la salud en personas no heterosexuales han criticado la ausencia de formación de profesionales, junto con la escasez de participación de colectivos LGBTI+ en el diseño de políticas públicas (Cabezas 2020; Estay, Valenzuela y Cartes 2020). Además, se ha señalado la vulneración persistente del principio de igualdad y no discriminación en la atención en salud (Basualto 2020) y cómo, en el diseño de políticas públicas, se mantiene un paradigma biomédico, lo que repercute negativamente en esta población (Cristi 2021). Otras investigaciones (Barrientos et al. 2019; Montecinos y Silva 2019; Morrison et al. 2019; Muñoz 2019) dan cuenta del impacto negativo de ello en la salud mental en esta población.

Este artículo se centra en el análisis de las políticas públicas de salud, de carácter universal, para visualizar la construcción del sujeto de esa política, sin incluir las normativas aplicadas a nivel local que se han ido desarrollando a través de los años ${ }^{2}$.

Durante los años de transición a la democracia en Chile (1990-2005), la retórica del consenso tuvo como uno de sus efectos la neutralización de la diferencia, obligando a la diversidad a ser no-contradicción (Richard 2010). Este panorama definirá la década de 1990 en torno a la postergación de las demandas LGBTI+ (Rivas 2011). Esto debido a que se planteaba que el país aún no se encontraba preparado para un cambio cultural de esa naturaleza (Garrido 2016). El mantenimiento de la sodomía como delito a través del artículo 365 del Código Penal, y la persecución a las personas trans bajo el amparo del artículo 373 de la Ley del Pudor, Moral y Buenas Costumbres, muestra cómo fueron criminalizadas durante los primeros años de la transición.

El "Programa de Identidad de Género" del Hospital Sotero del Rio desde 2018; el "Policlínico de Identidad de Género" del Hospital El Pino desde 2018; el "Policlínico de Identidad de Género" del Hospital Carlos Van Buren desde 2015; "Casa Trans" perteneciente al sindicato Amanda Jofré (Pinto 2019). El Hospital Las Higueras desde 2012, a través de la "Mesa de Participación en Salud para Mujeres Lesbianas y Bisexuales del Servicio de Salud Talcahuano"; el "Policlínico Trans" y el "Protocolo de Atención y Derivación Para Usuarios/as Trans" (SST s/a, 5). Prestan apoyo entidades Todo Mejora, Acción Gay, Juntos Contigo Centro de Salud amigable de MUMS, área de salud de Organizando Transdiversidades (OTD) (La Tinta 2019). También se han desarrollado guías en el ámbito de atención primaria de la salud (Montero y González 2017) y en disciplinas como la medicina (Valenzuela y Cartes 2019), la psicología (Martínez et al. 2018) y la terapia ocupacional (Morrison et al. [en prensa]). 
Posteriormente los gobiernos se ven interpelados no sólo por los movimientos LGBTI+, sino también por la necesidad del Estado de visibilizarse como una nación moderna y respetuosa de los DDHH, buscando estrategias para atender la agenda progresista internacional que comenzaba a instalarse (Galaz et al. 2018). Esta presión internacional se ha intensificado en los últimos años con nuevas consideraciones en torno a la identidad de género a partir de los cambios en la clasificación diagnóstica de manuales como el DSM IV y el CIE-10 (Robles y Ayuso-Mateos 2019). Como menciona Amigo-Ventureira (2019), la despatologización de las identidades trans es uno de los principales focos de lucha en relación con el ámbito de la salud actual. Se menciona que las modificaciones en las legislaciones han tensionado a la labor médica tradicional ya que las personas piden un mayor involucramiento en lo que respecta a la atención (Farji 2018). Meske y Antoniucci (2021) recalcan que esto abre nuevos sentidos a las categorías de sexo y género dentro de las legislaciones. Cox y Carrasco (2020) mencionan que el concepto de identidad de género y la forma que se entiende la relación sexo-género dentro de un equipo médico-terapéutico está resultando fundamental en relación con la atención de niños/as y adolescentes.

\section{Saberes dominantes en salud}

El campo de las sexualidades emerge como problemático, pues los saberes dominantes psi, médicos, jurídicos y religiosos han contribuido a la consolidación de categorías dicotómicas que van desde lo normal/anormal, hetero/homo, masculino/femenino, patológico/sano. Las sexualidades van reglándose por medio de una serie de discursos públicos, explícitos y sutiles (Wittig 1992). Muchas de estas construcciones sociales se basan en saberes biológicos reduccionistas que eliminan las posibilidades de pensar a los cuerpos sexuados como productos de contextos sociohistóricos específicos (Romero, García y Bargueiras 2005).

Desde esas disciplinas se fundamentaron comprensiones y teorías psiquiátricas que establecieron a la homosexualidad como enfermedad mental: desde 1952, con la publicación del Manual Diagnóstico y Estadístico de los Trastornos Mentales (DSM), de la Asociación Estadounidense de Psiquiatría (APA), se le clasificó como una enfermedad psiquiátrica (APA 1952). Dejó de ser considerada como una patología en 1973, con la segunda versión del DSM, reemplazándose por "perturbaciones en la orientación sexual" en el DSM-II y posteriormente por "homosexualidad egodistónica" en el DSM-III, que se eliminaría en 1986. La OMS eliminó a la homosexualidad como patología en 1990, producto de la presión social de organizaciones civiles, y en el año 2018 señaló que las personas trans no responden a una patología (Cáceres, Talavera y Reynoso 2013). La OMS en 2018 pasó la transexualidad del capítulo de "trastornos" al de "condiciones relativas a la salud sexual", pasando a llamarse incongruencia de género. Parte del argumento sobre la manten- 
ción de un criterio diagnóstico para las personas trans radica en el desarrollo de políticas públicas sanitarias que permitirían la intervención médica desde una evaluación ad hoc con características biológicas y psicológicas. De esta manera, el diagnóstico se plantea como una forma de asegurar un derecho (Borraz 2017). De ahí la importancia de auscultar los saberes médicos, pues no se constituyen exclusivamente en la especialización de sus conocimientos, sino que toman posición en un conjunto de relaciones sociales que implican acciones sobre ciertos sujetos. A pesar de ello, se constituyen maniobras de resistencia o estrategias de construcción/deconstrucción crítica (Lorente 2002). Así, emergen diversos colectivos exigiendo derechos sexuales (Preciado 2002), generando resistencias a saberes dominantes que buscan la regulación de sus prácticas y cuerpos (Foucault 1977).

\section{Marco metodológico}

Siguiendo a Carrasco, López y Estay (2012), empleamos el análisis documental para identificar, dentro de los textos, categorías discursivas que fuesen significativas para ver cómo se construye la noción de diversidad sexual en diferentes políticas públicas en relación con la salud. En una primera etapa, se desarrolló una sistematización de las normas generales aplicadas en el Estado chileno en materia de educación y salud en relación con género y sexualidad. De este catastro inicial que concitó 70 documentos, se seleccionaron 11 de las carteras ministeriales de salud y educación que resultarán más pertinentes en relación a la inclusión en salud de personas LGBTI+ bajo tres criterios: a) rescatando aquellos que fueron difundidos a nivel nacional en relación a la educación sexual; b) algunos que explícitamente nominaran a las personas no heterosexuales como parte de la norma; y c) que fueran de carácter normativo o propositivo a nivel general del país y no programas de implementación. En una segunda etapa, se sistematizó la información, considerando el tipo de sujeto-objeto de la política que subyacía a la norma y la forma de nominación y visibilización de las personas no heterosexuales en dichos documentos. Siguiendo un análisis discursivo, construimos categorías en base a la articulación temática del discurso (Sayago 2014). 
Tabla 1.

Corpus de análisis: programas y guías sobre salud y sexualidad

\begin{tabular}{|c|c|c|}
\hline Tipo & Nombre & Unidad \\
\hline Informe & $\begin{array}{l}\text { Adolescentes, sexualidad y embarazo: Perspecti- } \\
\text { vas desde la atención primaria de salud }\end{array}$ & Ministerio de Salud \\
\hline Informe & Programa Sexualidad Responsable & Ministerio de Salud \\
\hline Guía & $\begin{array}{l}\text { Hacia una sexualidad responsable: modelo de } \\
\text { gestión local }\end{array}$ & $\begin{array}{l}\text { Ministerio de Educación } \\
\text { de Salud, de la Mujer }\end{array}$ \\
\hline Ley & $\begin{array}{l}\text { Ley } \mathrm{n}^{\mathrm{O}} \text { 20.418. normas sobre información, orienta- } \\
\text { ción y prestaciones en materia de regulación de } \\
\text { la fertilidad }\end{array}$ & Ministerio de Salud \\
\hline Via Clínica & $\begin{array}{l}\text { Via clínica para la adecuación corporal en perso- } \\
\text { nas con incongruencia entre sexo fisico e identi- } \\
\text { dad de género }\end{array}$ & Ministerio de Salud \\
\hline Circular & $\begin{array}{l}\text { Circular n. }{ }^{\circ} 34 \text { sobre la atención corporal en } \\
\text { personas trans y fortalecimiento de la estrategia } \\
\text { de Hospital Amigo a personas de la Diversidad } \\
\text { Sexual }\end{array}$ & Ministerio de Salud \\
\hline Programa & $\begin{array}{l}\text { Programa Nacional de Salud Integral de Adoles- } \\
\text { centes y Jóvens }\end{array}$ & Ministerio de Educación \\
\hline Circular & $\begin{array}{l}\text { Circular } n^{\circ} 21 \text {. instrucción sobre la atención de } \\
\text { personas trans en la red asistencial }\end{array}$ & Ministerio de Salud \\
\hline Circular & $\begin{array}{l}\text { Circular } \mathrm{n}^{\circ} 18 \text {. sobre ciertos aspectos de la aten- } \\
\text { ción de salud a niños y niñas intersex }\end{array}$ & Ministerio de Salud \\
\hline Circular & $\begin{array}{l}\text { Complementa circular } \mathrm{n}^{\circ} 18 \text {. sobre ciertos aspectos } \\
\text { de la atención de salud a niños y niñas intersex }\end{array}$ & Ministerio de Salud \\
\hline Guía & $\begin{array}{l}\text { Consejería en salud sexual y reproductiva para } \\
\text { adolescentes }\end{array}$ & Ministerio de Salud \\
\hline
\end{tabular}

Fuente: Elaboración propia

\section{Resultados y discusión}

Se rastrearon tres formas en que se condensa la actuación del Estado en materia de salud hacia la población LGBTI+ desde el fin de la dictadura: en un primer momento, una cierta invisibilidad como norma; en un segundo momento, una construcción problemática de la orientación sexual e identidad de género; y por último, en los últimos años, la sobre-especificación identitaria de la política pública en salud. 


\subsection{La invisibilidad como norma o la "política de la no política"}

En este primer apartado vemos cómo la invisibilidad de las personas LGBTI+ en políticas públicas no es algo considerado inocuo porque esa propia opacidad es una política en sí misma. Es decir, en más de 10 años de políticas de salud en democracia, las personas LGBTI+ fueron invisibles, pese a la consolidación de los diversos movimientos sociales en el país.

En el año 1992, en el estudio "Adolescentes, sexualidad y embarazo: Perspectivas desde la atención primaria de salud" (Marshall 1992), se plantea esta situación y se visualiza cómo los equipos de salud de la atención primaria, enfocados en la población adolescente, no consideran las experiencias de personas LGBTI+. En dicho informe se destaca que sólo se concibe en salud sexual elementos como embarazo, prevención de enfermedades de transmisión sexual y anticoncepción (Marshall 1992). Esta perspectiva heterosexual y de riesgo de la intervención en salud se mantiene durante todo el primer tramo de democracia. Así se evidencia también en el "Programa de sexualidad responsable" (MINSAL 2002), donde el foco está en la prevención del embarazo adolescente y de infecciones de transmisión sexual entre parejas heterosexuales.

Esta invisibilización de las experiencias LGBTI+ vuelve a emerger en el informe "Chile: Situación de la salud y los derechos sexuales y reproductivos". En dicho documento se omite información sobre las problemáticas, necesidades y experiencias asociadas a las personas LGBTI+ en los centros de salud. Esta invisibilidad vuelve a presentarse en el documento orientativo interministerial "Hacia una sexualidad responsable: modelo de gestión local". Este modelo nace con

[...] la tarea de abordar la sexualidad de sus usuarios, focalizando su accionar en la salud sexual, entendida como el proceso centrado en el desarrollo humano y en las relaciones interpersonales, y no solamente en la atención al ámbito reproductivo, las enfermedades de transmisión sexual y la prevención de riesgos. (MINEDUC, MINSAL y Ministerio de la Mujer 2004)

Se refuerza la idea de los derechos y deberes en sexualidad, como la entrega de información, confidencialidad y estilos de vida saludables. Sin embargo, siempre el enfoque es desde un posicionamiento binario y heterosexual y no se especifican acciones u orientaciones en salud mental, ginecología o infecciones de transmisión sexual respecto de personas que no sean heterosexuales.

Esta consideración del ideal heterosexual como sujeto universal de la política pública se basa en lo que Mulé et al. (2009) han llamado una "ideología de la heterosexualidad". Esta ideología promovería una "no especificidad", lo que permite que las necesidades de las personas no heterosexuales queden invisibilizadas, a la vez que desde las políticas públicas pareciera que discursivamente se incluye a toda la ciudadanía. Así, las normativas en torno a la salud sexual naturalizan la heterosexualidad, institucionalizando la discriminación como fundamento de la política. 


\subsection{La construcción problemática de la orientación sexual e identidad de género}

Entre los años 2000 y 2015, los movimientos LGBTI+ logran visibilizar la dimensión política de la sexualidad, transitando desde el ámbito privado hacia lo público (Schenck 2018). Las políticas públicas en esta materia se centran en la salud sexual, pero poniendo un acento en el control de ciertas enfermedades. Aunque en este periodo se posibilitan medidas antidiscriminatorias, derechos colectivos no considerados hasta la fecha para personas LGBTI+ y hacer más pertinentes algunos servicios, a la vez se configura un dispositivo de regulación fundamentado en la consideración de las comunidades LGBTI+ como "población de riesgo". Esto se lleva a cabo por cómo se vinculan las vivencias no heterosexuales y los cuidados respecto de enfermedades sexuales, contribuyendo no solo al despliegue de servicios, protocolos y atenciones en esta línea, sino también a la concreción de un imaginario estereotipado de cuerpos LGBTI+ y la propagación de enfermedades. Por ejemplo, en el "Reglamento sobre Infección de Transmisión Sexual" (MINSAL 2011a), que establece instancias de educación en la materia, se da cuenta de la diversidad sexual y de género como uno de los aspectos importantes a tener en consideración. Asimismo, se crea el "Programa Nacional de prevención y control de VIH/SIDA y las ITS" (MINSAL 2011b) que destaca, dentro de la noción de "vulnerabilidad", explícitamente a la población LGBTI+, señalando que:

[...] en un análisis de vulnerabilidad frente al VIH y las ITS no solo tendrá relevancia la discriminación por orientación sexual o actividad (discriminación a hombres homo-bisexuales, hombres que tienen sexo con hombres o a mujeres que tienen sexo con mujeres, personas que ejercen el comercio sexual), sino también las discriminaciones de género, de etnia, de nivel socioeconómico. (MINSAL 2011b, 8)

En varias oportunidades se menciona en esta disposición que los programas de salud van especialmente dirigidos a la comunidad no heterosexual, lo que, siendo una posibilidad de atención para estas personas, contribuye indirectamente a que sea vista la transmisión de enfermedades sexuales como una problemática casi exclusiva de estos colectivos. Esta asociación negativa intenta ser moderada a partir del año 2013, cuando se elimina la norma general técnica $n^{\circ} 0146$ que prohibía a homosexuales ser donantes de sangre (MINSAL 2013). Este hecho intenta desnaturalizar el vínculo entre enfermedades de transmisión sexual y personas no heterosexuales.

En el Programa Nacional de Salud Integral de Adolescentes y Jóvenes de 2012 se amplía a otras experiencias de la población juvenil (MINSAL 2011a). Se destaca en el documento que: "falta abrir la discusión a temas como aborto, homosexualidad y equidad de género", agregando que se requiere "abrir discusión a temas tabú (aborto terapéutico, homosexualidad, transgénero)" (MINEDUC 2012, 149). Si bien emerge como un nuevo campo que requiere una intervención de 
salud desde un punto de vista integral, no se especifican propuestas específicas respecto de las experiencias LGBTI+.

Asimismo, se crea la "Consejería en Salud Sexual y Reproductiva para Adolescentes" (MINSAL 2015b), la cual entrega lineamientos para profesionales de atención primaria que trabajan con adolescentes LGBTI+ y heterosexuales. Dicha guía nace en respuesta al programa Nacional de Salud para Adolescentes y Jóvenes del año 1995 (MINSAL 1995). En el apartado de conceptualizaciones, se señalan elementos relacionados con personas LGBTI+, pero no se profundiza mayormente en elementos constitutivos de la identidad de género, ni de problemáticas asociadas a cada grupo en particular.

Solo en el año 2015 emerge una visión pública donde se consideran espacios específicos en salud, respecto de la orientación sexual e identidad de género. En la "Guía Práctica: Consejería en Salud Sexual y Reproductiva para Adolescentes" (MINSAL 2015b), se incluyen conceptos como: salud sexual y reproductiva; consejería para la prevención del embarazo y enfermedades de transmisión sexual; uso de preservativos; abordaje de distintas orientaciones sexuales e identidades de género. En esta guía se ponen en relevancia los conceptos asociados a las experiencias LGBTI+ en relación con la salud sexual, abriendo consejerías específicas. Pero el documento enfatiza que entre su público objetivo se sitúan usuarios/as "adolescentes que presentan dudas o confusión en la orientación sexual, en su identidad de género o adolescentes que se definan gay, lesbianas, bisexuales o trans" (MINSAL 2015b, 13). Dentro de los temas que se proponen para las consejerías están:

[...] explorar la conducta sexual y clasificar grados de Petting, que señalan el tipo de intimidad sexual (...). Cuando pregunte por la orientación sexual incluya las opciones: "heterosexual", "homosexual", "bisexual", "prefiero identificarme como...", de esta manera se trabaja con un enfoque inclusivo de la diversidad sexual (...). No asuma que es heterosexual, homosexual o bisexual. (MINSAL 2015b, 21)

Asimismo, se plantea el libre ejercicio de la personalidad de los/as adolescentes, enfatizando su derecho a la sexualidad, sin ser discriminados por su orientación sexual. También los mecanismos de protección como preservativo femenino y masculino emergen como claves en las consejerías "en todas las prácticas sexuales, considerando a la diversidad sexual" (MINSAL 2015b, 33).

En dicho documento existe un apartado específico para enfrentar dudas sobre orientación sexual e identidad de género, entre las que se destacan: el conocimiento de creencias y actitudes frente a personas LGBTI+; no asumir la heterosexualidad como base; la importancia de no señalar la homosexualidad como enfermedad; que las dudas sobre orientación sexual no requieren tratamiento médico; las formas de acompañamiento más directo; material educativo; la importancia de la derivación cuando el/la profesional considere que tiene prejuicios sobre personas LGBTI+, entre otras. Finalmente, dicho texto culmina con un glosario referente a las concep- 
tualizaciones que deben comprenderse para la atención de personas no heterosexuales. Al respecto, es interesante mencionar que se aborda la noción de diversidad sexual como un todo aglutinante sin especificar las necesidades específicas de personas lesbianas, bisexuales, homosexuales, trans o intersex. No obstante, se aclara que pertenecer a este conglomerado no implica obligatoriamente una necesidad de tratamiento médico específico y se hace un llamado de atención a considerar la homofobia, transfobia y heterosexismo como elementos dentro de las prácticas de atención en salud que pueden producir vivencias conflictivas en las personas.

\subsection{La sobre-especificación identitaria de la política pública}

Finalmente, en los últimos años se ha dado una cierta hipervisibilización desde la intervención pública sobre ciertos cuerpos no heterosexuales, especialmente de las personas trans. Por un lado, esto resulta positivo porque posibilita que este colectivo pueda acceder al ámbito de salud, después de muchos años de invisibilización. Pero, por otro, se corre el riesgo de una sobre-intervención basada en nuevos estereotipos sobre estos cuerpos, nuevos procesos de psicologización y patologización y distinciones en la atención respecto de otros colectivos no heterosexuales.

En los últimos años, han emergido circulares y normativas institucionales específicas sobre las personas trans e intersex. En el año 2010 se crea la "Vía Clínica para la adecuación corporal en personas con incongruencia entre sexo físico e identidad de género" (MINSAL 2010). En dicho documento se especifican las intervenciones pertinentes, sus objetivos, criterios de elegibilidad y disposición para su preinscripción. Para poder acceder a este tipo de prestación, el ministerio señala una problemática que debe ser atendida, definiéndola como "la persistencia de un sentimiento de incongruencia entre sexo físico e identidad de género que cause incomodidad, estrés o impedimento significativo en cualquier área de su funcionamiento personal"' (MINSAL 2010, 5).

Es relevante señalar que las personas que deseen realizar este procedimiento deben ser mayores de 18 años. El proceso formal que estipula la norma considera tres factores: salud mental, adecuación hormonal y adecuación quirúrgica. El documento establece una evaluación inicial de un psicólogo/a, el posible desarrollo de una psicoterapia, seguimiento y confirmación por parte del especialista-médico para otro tipo de intervenciones. Luego, en el proceso de adecuación hormonal se explican los factores asociados al consumo de estos medicamentos y sus efectos primarios y secundarios. Finalmente, el proceso puede - o no - terminar en una adecuación quirúrgica. Para esto se establece que se debe cumplir con ciertos criterios de elegibilidad: haber realizado una psicoterapia, tratamiento hormonal, conocimiento demostrado de riesgos de la operación (MINSAL 2010).

En el documento quedan pendientes temas relevantes como la dificultad en el acceso de personas menores de 18 años, y también el deseo de personas que no 
quieran efectivamente llevar a cabo el proceso completo de tratamiento. Esto evidencia cierta visión normativa y adultocéntrica respecto de los márgenes aceptables para definir la identidad de género y el sexo congruente con esta misma. Por otra parte, la continuidad del acompañamiento post operatorio es inexistente.

En ese sentido, el dispositivo sanitario opera principalmente para personas mayores que supuestamente son "conscientes" de la incongruencia que implica la experiencia trans y desean un cambio corporal completo. Así, mientras más consciencia, mayor será el nivel de cambio al que se le permitirá acceder. En este documento el concepto de incongruencia opera como marco de referencia de lo trans, regulando a nivel nacional los procedimientos de adecuación corporal según este principio. Esto aunque en la Vía Clínica se plantea que "la psicoterapia no intenta 'curar' la incongruencia (...) la psicoterapia es un criterio de elegibilidad para la intervención hormonal y quirúrgica” (MINSAL 2010, 5).

Mientras que se abandona una mirada tradicional de la "cura", ya que no se intenta cambiar de parecer a la persona, sino que lograr que la persona acepte la identidad de género y sexo físico "congruente", se establece una nueva noción que es la "aceptación de la discordancia", reafirmando que lo normal es la correspondencia entre identidad y sexo físico. Bajo la advertencia de "irreversibilidad" de la intervención biológica final, que pasaría a ser la culminación del tránsito, se insta al sujeto a asumir responsabilidad a propósito de su propia identidad. De esta forma, implícitamente se establece que el abanico de posibilidades de vivencias trans se encuentran erradas, por lo que sería necesario cierto sistema de intervención y normalización que elimina la posibilidad de inestabilidad e incoherencia en las categorías (Echeverría y Maturana 2016).

En tanto, para las personas intersexuales se lanza una circular del año 2015 con la que se instruye cierto freno a las intervenciones quirúrgicas a recién nacidos/as y niños/as intersex (MINSAL 2015a, 2016), la cual se ampara en las recomendaciones de la OMS y la Declaración Internacional de los Derechos del Niño. A pesar de ser un aporte contra los casos de intervención temprana, no cuestiona la asignación de sexo registral, la cual se lleva a cabo bajo una lectura médica respecto de "las mejores expectativas" que se proyecten para el desarrollo del cuerpo, a través de exámenes interdisciplinarios. En ese sentido, se obvian los riesgos y daños que esta decisión realizada desde la mirada experta puede provocar en la persona en términos del desarrollo de su identidad de género.

\section{Conclusiones}

La gestión pública de salud en torno a las personas LGBTI+ durante la postdictadura ha cruzado cambios significativos asociados a la transformación de la sociedad en relación con la mayor apertura cultural sobre estas vivencias. Este camino ha implicado un cierto desplazamiento de la noción aglutinante de "diver- 
sidad sexual" como problema y amenaza - relacionada a regulaciones sobre esos cuerpos - a una concepción más abierta y de reconocimiento. Pero las principales acciones se han enfocado en base a la mantención de imaginarios normativos de los cuerpos y vinculados a valores liberales como el derecho individual a la salud, la responsabilidad individual y la instauración de procesos psico-médicos - a partir de diagnósticos físicos y psicológicos - que garantizan el acceso a ciertos derechos. Por tanto, más allá de transformar los marcos de sentido con los que opera el ámbito de la salud, reifican cierto disciplinamiento de los sujetos a costa de su inclusión. Asimismo, en general se puede trazar una acción del Estado que ha tenido un carácter sectorial y parcial, sin plantearse una política pública transversal que ampare los derechos de las personas LGBTI+.

Es relevante mencionar que, en los últimos cinco años, se ha producido un proceso incipiente de institucionalización en torno a la identidad de género en distintos niveles administrativos (espacios clínicos o unidades de identidad de género y también circulares y normas específicas). No obstante, la implementación de disposiciones, circulares y normativas quedan al arbitrio de las instituciones por las que circulan, sin implementar necesariamente mecanismos para su ejecución. Es importante mantener la mirada crítica, a propósito de esta institucionalización, de cómo operan los discursos de la diversidad sexual, ya que puede generar nuevos dispositivos de normalización y regulación, los que, si bien pueden garantizar el acceso a salud, no necesariamente consideran a las personas LGBTI+ como sujetos plenos de derecho. En ello, los procesos de "reflexividad crítica" sobre las propias prácticas y discursos de los/as agentes de la salud como la "vigilancia epistemológica" referida a los preconceptos que se tienen de la diversidad sexual en la atención (Galaz y Rubilar 2019) resultan fundamentales para poder cumplir con el principio de no dañar a las personas usuarias y buscar los mejores caminos de resolución médica frente a los/as pacientes, independiente de su orientación o identidad de género. En ese sentido, es importante integrar a la misma población LGBTI+ en el proceso del diseño de políticas públicas (Cabezas 2020; Cristi 2021), además de ir de la mano de la formación de funcionarios/as que trabajan con esta población. Pero para ello se requiere a la vez cuestionar la mirada individualista, asistencialista y biologicista, integrando lógicas participativas que consideren las necesidades manifestadas desde los propios colectivos LGBTI+, y no sólo a partir del saber experto.

\section{Agradecimientos}

Proyecto Fondecyt N 1170417 “Acción pública y diversidad sexual en Chile: Construcciones sociales en democracia (1990-2016)” (CONICYT-Chile). 


\section{Referencias bibliográficas}

APA - American Psychiatric Association. 1952. Mental Disorders. Diagnostic and Statistical Manual. Washington: APA.

Amigo-Ventureira, Ana. 2019. "Un recorrido por la historia trans*: desde el ámbito biomédico al movimiento activista-social." Cadernos Pagu 57: 1-26. DOI:10.1590/18094449201 900570

Barrientos, Jaime, José Saiz, Fabiola Gómez, Mónica Guzmán-González, Ricardo Espinoza-Tapia, Manuel Cárdenas, y Joaquín Bahamondes. 2019. “La Investigación Psicosocial Actual Referida a la Salud Mental de las Personas Transgénero: Una Mirada Desde Chile." Psykhe 28: 1-13. DOI:10.7764 / psykhe.28.2.1482

Basualto, Marcelo. 2020. "Libertad religiosa y no discriminación en prestación de servicios a personas LGBTI. Hacia algunos criterios de solución en Chile que cumplan estándares de derecho internacional de derechos humanos." Tesis Magíster, Universidad Diego Portales.

Borraz, Marta. 2017. "La OMS deja de considerar la transexualidad un trastorno mental." El Diario, 31 enero. Disponible en https:/ / www.eldiario.es/ sociedad/OMS-considerar-transexualidad-enfermedad-incongruencia_0_783572396.html

Cabezas, Yasna. 2020. “Características de la atención de salud efectiva para la población que se identifica con el colectivo LGBTI." Tesis Magíster, Pontificia Universidad Católica de Chile.

Cáceres, Carlos, Víctor Talavera, y Rafael Reynoso. 2013. “Diversidad sexual, salud y ciudadanía." Revista Peruana de Medicina Experimental y Salud Pública 30(4): 698-704. DOI:10.17843/rpmesp.2013.304.255

Carrasco, Claudia, Verónica López, y Camilo Estay. 2012. “Análisis crítico de la Ley de Violencia Escolar de Chile." Psicoperspectivas 11(2): 31-55. DOI: 10.5027 / psicoperspectivas-Vol11-Issue2-fulltext-228

Cox, Paula, y María Carrasco. 2020. “Disforia de género en niños y controversias en su tratamiento: dos concepciones distintas sobre la identidad de género." Persona y bioética 24: 57-76. DOI: $10.5294 /$ pebi.2020.24.1.5

Cristi, Daniela. 2021. "Políticas de identidad y gestión sexual de los cuerpos: un análisis de los discursos legislativos acerca de la gestión estatal del conflicto trans en Chile." La ventana. Revista de estudios de género 6(53), 276-312. DOI:10.32870/lv.v6i53.7192.

Echeverría, Genoveva, y José Maturana. 2016. "Análisis crítico del discurso de políticas públicas en diversidad sexual en Chile." Universitas Psychologica 14(4): 1485-1498. DOI: 10.11144/Javeriana.up14-4.acdp

Esguerra, Camilla, y Jaison Bello. 2014. “Interseccionalidad y políticas públicas LGBTI en Colombia: usos y desplazamientos de una noción crítica." Revista de Estudios Sociales 49: 19-32. DOI:10.7440/res49.2014.02

Estay, Fernanda, Amanda Valenzuela, y Ricardo Cartes. 2020. “Atención en salud de personas LGBT+: Perspectivas desde la comunidad local penquista." Revista Chilena de Obstetricia y Ginecología 85: 351-357. DOI: 10.4067/S0717-75262020000400351

Farji, Anahí. 2018. “Los/as profesionales de la salud frente a la Ley de Identidad de Género argentina. Tensiones entre el saber experto y el cuidado integral." Physis: Revista de Saúde Coletiva 3(28): 1-18. DOI: 10.1590/ s0103-73312018280318

Foucault, Michel. 1977. Historia de la sexualidad I: La voluntad de saber. Madrid: Siglo XXI.

Galaz, Caterine, Mauricio Sepúlveda, Rolando Poblete, Lelya Troncoso, y Rodolfo Morrison. 2018. "Derechos LGBTI en Chile: tensiones en la constitución de otredades 
sexualizadas." Psicoperspectivas. 17 (1): 6-16. DOI: 10.5027/ psicoperspectivas-vol17issue1-fulltext1165

Galaz, Caterine, y Graciela Rubilar. 2019. “Experiencias profesionales en intervención psicosocial: el ejercicio narrativo como metodología de reflexividad y vigilancia epistemológica." RELMECS 9(1), e050. DOI: 10.24215/18537863e050

Garrido, Juan. 2016. "Memoria colectiva, discursos y violencia homo-lesbotransfóbica en la dictadura militar y transición democrática en Chile." Documento de Trabajo $\mathrm{N}^{\circ} 24$, Santiago: ICSO, Universidad Diego Portales. Disponible en https: / / www.researchgate.net/publication/316993668_Memoria_colectiva_discursos_y_violencia_homo-lesbotransfobica_en_la_dictadura_militar_y_transicion_democratica_en_Chile

Iosa, Tomás, y Hugo Rabbia. 2011. "Definiciones divergentes de la estrategia de visibilidad en el movimiento LGTB cordobés." Íconos. Revista de Ciencias Sociales 39: 61-77. DOI: 10.17141/iconos.39.2011.743

La Tinta. 2019. "El avance de consultorios inclusivos." La Tinta, 6 noviembre. Disponible en https: / / latinta.com.ar/2019/11 / el-avance-de-consultorios-inclusivos /

Lorente, Belén. 2002. "Trabajo social y ciencias sociales. Poder, funcionalización y subalternidad de saberes." Trabajo Social 4: 41-60. Disponible en https: / / revistas.unal.edu.co/ index.php/tsocial/ article/view/32516

Marshall, Teresa. 1992. Adolescentes, sexualidad y embarazo: Perspectivas desde la atención primaria de salud. Santiago: Iberoamericana.

Martínez, Claudio, Alemka Tomicic, Constanza Gálvez, Juliana Rodríguez, Catalina Rosenbaum y Francisco Aguayo. 2018. Psicoterapia culturalmente competente para el trabajo con pacientes LGBT+: una guía para psicoterapeutas y profesionales de la salud mental. Santiago: Universidad Diego Portales.

Meske, Verónica, y Melina Antoniucci. 2021. "El sexo en disputa: regulación y materialización corporal del género en un contexto de despatologización de la identidad." Historia y Sociedad 40: 198-223. DOI: 10.15446 / hys.n40.86873

Mesa de Participación en Salud para Mujeres Lesbianas y Bisexuales. (s/a). Servicio de Salud Talcahuano. Disponible en https:/ / sstalcahuano.cl/salud-y-diversidad-sexual/\#1529 678576814-31ef86a3-6682

Ministerio de Educación [MINEDUC]. 2012. Programa Nacional de Salud Integral de Adolescentes y Jóvenes. Gobierno de Chile.

Ministerio de Educación, Ministerio de Salud [MINSAL] y Ministerio de la Mujer. 2004. Hacia una sexualidad responsable: modelo de gestión local. Santiago: Ed. Ikastola.

Ministerio de Salud. 1995. Programa Nacional de Salud para Adolescentes y Jóvenes. Gobierno de Chile.

Ministerio de Salud. 2002. Programa Sexualidad Responsable. Gobierno de Chile.

Ministerio de Salud. 2010. Vía clínica para la adecuación corporal en personas con incongruencia entre sexo físico e identidad de género. Gobierno de Chile.

Ministerio de Salud. 2011a. Reglamento sobre infecciones de transmisión sexual. Gobierno de Chile.

Ministerio de Salud. 2011b. Programa Nacional de prevención y control de VIH/SIDA y las ITS. Gobierno de Chile.

Ministerio de Salud. 2013. Aprueba Norma General Técnica $N^{\circ} 0146$, Nominada "Norma que regula el procedimiento de atención de donantes de sangre (en sitio fijo o móvil).» Gobierno de Chile.

Ministerio de Salud. 2015a. Circular no 18. Instruye sobre ciertos aspectos de la atención de salud a niños y niñas intersex. Gobierno de Chile. 
Ministerio de Salud. 2015b. Guía práctica: Consejería en salud sexual y reproductiva para adolescentes. Orientaciones para los equipos de Atención Primaria. Gobierno de Chile.

Ministerio de Salud. 2016. Complementa circular no 18 que instruye sobre ciertos aspectos de la atención de salud a niños y niñas intersex. Gobierno de Chile.

Montecinos, María José, y Ana María Silva. 2019. “Percepción de personas de orientación homosexual respecto del enfoque hacia la diversidad sexual utilizado en Atención Primaria de Salud." Revista Chilena de Salud Pública 23(1): 60-67. DOI: 10.5354/07195281.2019.55050

Montero, Adela, y María Isabel González. 2017. Guía de orientaciones y recomendaciones para la atención de adolescentes y jóvenes gays, lesbianas y bisexuales en el nivel primario de salud. Universidad de Chile.

Morrison, Rodolfo, Lilian Araya, Tamara Palomino, y José Manuel Graus. 2019. "Situación de las niñas y niños trans en Chile: derechos humanos y políticas públicas." RELIES: Revista del Laboratorio Iberoamericano para el Estudio Sociohistórico de las Sexualidades 1: 26-48. Disponible en https: / / www.upo.es/revistas/index.php/relies/article/view/3842

Morrison, Rodolfo, Tamara Palomino-Araneda, Pía Muñoz-Coronado, y Nicole TapiaHenríquez. [en prensa]. “Experiencia de formación con comunidades educativas para la inclusión de estudiantes LGBTIQ+ desde un enfoque de derechos humanos." En Niñez, juventudes y derechos: Reflexiones a partir de prácticas de terapia ocupacional en ámbitos de salud mental, editado por M. J. Poblete, S. Jones, D. Grandón y P. Olivares, 1-24. Santiago: Editorial Universitaria.

Mulé, Nick, Lori Ross, Barry Deeprose, Beth Jackson, Andrea Daley, Ana Travers, y Dick Moore. 2009. "Promoting LGBT health and wellbeing through inclusive policy development." International Journal for Equity in Health 8(18). DOI: 10.1186/14759276-8-18

Muñoz, Carmen. 2019. La salud en Chile: Una historia de movimientos, organización y participación social. Valdivia: Ediciones Universidad Austral de Chile.

Pecheny, Mario. 2005. "Identidades discretas." En Identidades, sujetos y subjetividades, compilado por Leonor Arfuch, 131-153. Buenos Aires: Prometeo.

Pinto, Camilo. 2019. "Inauguran primera Casa Trans de Chile." El Desconcierto, 13 abril. Dispible en https: / /www.eldesconcierto.cl/nacional/2019/04/13/inauguran-primeracasa-trans-de-chile.html

Preciado, Paul B. 2002. Manifiesto contra-sexual. Madrid: Opera Prima.

Richard, Nelly. 2010. Crítica de la Memoria (1990-2010). Santiago: Univ. Diego Portales.

Rivas, Felipe. 2011. De la homosexualidad de Estado a la Disidencia Sexual. Santiago: CUDS.

Robles, Reveca, y Jose Ayuso-Mateos. 2019. “CIE-11 y la despatologización de la condición transgénero." Revista de Psiquiatría y Salud Mental 12(2): 65-67. DOI: 10.1016/j.rp sm.2019.01.002

Romero, Carmen, Silvia García, y Carlos Bargueiras. 2005. El eje del mal es heterosexual: figuraciones, movimientos y prácticas feministas «queer». Madrid: Traficantes de sueños.

Sayago, Sebastián. 2014. "El análisis del discurso como técnica de investigación cualitativa y cuantitativa en las ciencias sociales." Cinta Moebio 49: 1-10. DOI:10.4067/S0717554X2014000100001

Schenck, Marcela. 2018. "Incorporación de la diversidad genérico-sexual en salud: claves teóricas para un modelo analítico." Revista de la Facultad de Derecho 45: 318-343. DOI: $10.22187 / \mathrm{rfd} 2018 \mathrm{n} 45 \mathrm{a} 12$

Servicio Nacional de la Mujer. 2003. Chile: Situación de la salud y los derechos sexuales y reproductivos. Gobierno de Chile. 
Valenzuela, Amanda, y Ricardo Cartes. 2019. “Perspectiva de género en la educación médica: Incorporación, intervenciones y desafíos por superar." Revista Chilena de Obstetricia y Ginecología 84(1): 82-88. DOI: 10.4067/S0717-75262019000100082

Wittig, Monique. 1992. El pensamiento heterosexual y otros ensayos. Madrid: Egales.

Caterine Galaz V. Académica de la Facultad de Ciencias Sociales de la Universidad de Chile. Coordinadora del Núcleo Diversidad y Género: abordajes feministas interseccionales.

Correo electrónico: cgalazvalderrama@uchile.cl

Catalina Martínez-Conde. MScs, psicóloga. Estudiante de Doctorado, Universidad Autónoma de Barcelona y Universidad de Chile.

Correo electrónico: cata.alvarezmc@gmail.com

Rodolfo Morrison. Terapeuta Ocupacional. Doctor en Lógica y Filosofía de la Ciencia, Universidad de Chile.

Correo electrónico: rodolfo.morrison@uchile.cl

Artículo recibido el 27 de Octubre de 2020 y aceptado para su publicación el 31 de Marzo de 2021. 